\title{
microRNA Regulation of Synaptic Plasticity
}

\author{
Neil R. Smalheiser and Giovanni Lugli \\ Department of Psychiatry and UIC Psychiatric Institute, MC912, University of Illinois at Chicago, \\ 1601 W. Taylor Street, Chicago, IL 60612, USA
}

Neil R. Smalheiser: neils@uic.edu

\section{Abstract}

microRNAs play an important role in regulating synaptic plasticity. For example, microRNAs target (and are targeted by) plasticity mediators such as CREB, MECP2, and FMRP. As well, specific microRNAs have been shown to be expressed within dendrites, where they regulate protein translation of targets mediating dendritic growth. Components of the RISC machinery have been implicated in long-term memory in Drosophila. Here, we review evidence from studies of adult mouse forebrain supporting a model wherein synaptic stimulation (above a threshold value) increases calcium within dendritic spines, activates calpain, and activates and releases dicer from the postsynaptic density. Dicer processes local pre-miRs into mature miRNAs that are incorporated into RISC complexes within or near the dendritic spine, and that bind available target mRNAs in the vicinity. These may repress protein translation under resting conditions, yet permit a phasic burst of translation to occur transiently following subsequent synaptic activity. Loaded RISC complexes that are not bound to local mRNAs may serve to bind and trap mRNAs that are being transported down dendrites. Thus, locally formed microRNAs may mark the location of previously activated synapses and perform a type of synaptic tagging and capture.

\section{Keywords}

Dicer; RISC; microRNA; Pre-miR; Postsynaptic densities; Learning; Synaptic plasticity

\section{Introduction}

Several lines of evidence suggest that microRNAs play an important role in regulating synaptic plasticity. For example, plasticity mediators such as CREB, MECP2, FMRP, and HuB are "hubs" that are regulated by many miRNAs and, in turn, regulate miRNAs (John et al. 2004; Vo et al. 2005; Wu and Xie 2006; Klein et al. 2007; Nomura et al. 2008). As well, specific microRNAs have been shown to be expressed within dendrites, where they regulate protein translation of targets mediating dendritic growth (Schratt et al. 2006; see also Wayman et al. 2008). Components of the RISC machinery have been implicated in long-term memory in Drosophila (Ashraf et al. 2006). Please see Kosik (2006) and the accompanying articles in this Special Issue for further reviews.

Studies in developing cultured neurons have suggested that mature miRNAs reach dendrites by a mixture of passive diffusion and active "piggybacking" onto target mRNAs that are being transported (Kye et al. 2007). Such a mechanism might occur in vivo as well. However, as we will show in this review, in adult mouse forebrain, a large and diverse

(C) Humana Press Inc. 2009

Correspondence to: Neil R. Smalheiser, neils@uic.edu. 
population of pre-miRs are not quickly processed into mature miRNAs in the neuronal cell body, but rather appear to be transported to dendrites where they are "held" within dendritic spines in close association with the postsynaptic density. Similarly, dicer and RISC components are locally expressed in close proximity to the pre-miRs, and appear to comprise a system for local activity-dependent formation of mature miRNAs and local regulation of protein translation. This is consistent with precedents in various cell types showing that specific pre-miRs can be expressed yet inhibited from further processing into mature miRNAs (reviewed in Smalheiser 2008a). As well, several stable primary miRNA gene transcripts (pri-miRs) have been shown to be expressed in the cytoplasm (e.g., BIC; Eis et al. 2005) and one pri-miR has been shown to be localized sub-synaptically at the rat neuromuscular junction (Velleca et al. 1994).

\section{Dicer Is Expressed in Synaptic Fractions and Enriched at PSDs}

Several years ago, we proposed that RNA interference (RNAi) pathways may have a physiological role in regulating long-term gene expression in the normal mammalian CNS (Smalheiser et al. 2001). Since all types of tiny RNAs known at the time are processed by the RNAse III enzyme dicer, we decided to raise a series of antibodies against the Cterminal epitope of dicer (as well as several internal epitopes) and characterize its expression in adult mouse forebrain. Dicer protein was predominantly expressed within large projection neurons in a somatodendritic pattern (Lugli et al. 2005). Furthermore, using immunocytochemistry at the electron microscopic level, dicer was detected within dendritic spines, particularly in association with the postsynaptic density (Fig. 1; Lugli et al. 2005).

Subcellular fractionation revealed that dicer was enriched both within synaptoneurosomes (consisting predominantly of pinched-off dendritic spines) and synaptosomes (consisting predominantly of presynaptic terminals with adherent synaptic cleft and postsynaptic densities), and was particularly enriched in isolated postsynaptic densities (Fig. 2a; Lugli et al. 2005, 2008).

Nevertheless, when isolated postsynaptic densities were examined for RNAse III activity using long exogenous double-stranded RNA as a substrate, no significant enzymatic activity was detected. At about this time, several laboratories reported that limited non-specific proteolytic cleavage of dicer (by proteinase K) greatly enhances RNAse III activity (Provost et al. 2002; Zhang et al. 2002). We reasoned that perhaps dicer at the postsynaptic density is cryptic under "resting" conditions and only activated following synaptic activity that causes activation of some critical protease(s). Since calpain is well known to play a role in synaptic plasticity, we tested whether calpain may cleave dicer and activate its RNAse III activity. Recombinant dicer is cut by calpain into a series of fragments, down to a stable $28 \mathrm{kDa}$ endfragment that contains the $\mathrm{C}$-terminus and that is highly active in processing doublestranded RNA into. $\sim 22 \mathrm{nt}$. products (Lugli et al. 2005). Furthermore, when isolated postsynaptic densities were incubated with calpain, dicer and discrete dicer fragments are released and RNAse III activity is greatly stimulated. We also obtained evidence that calpain cleaves dicer in living tissue. Exposing synaptoneurosomes to elevated calcium in the medium, or treating hippocampal slices with NMDA (50 microM, $15 \mathrm{~min}$ ) causes the appearance of a discrete $75 \mathrm{kDa}$ fragment of dicer in a calcium- and calpain-dependent manner (Lugli et al. 2005).

\section{What Is the Substrate for Dicer?}

These studies indicate that dicer protein is expressed near synapses and that synaptic activation can stimulate its RNAse III activity and its release from the postsynaptic density. But what is the local substrate for dicer? One possibility is that dicer is not acting in its role as part of the RNA interference pathway, but instead has a role related to its interactions 
with 5-lipoxygenase (Dincbas-Renqvist et al. 2009). Another possibility is that when dicer is released from postsynaptic densities, it becomes translocated from synapses to the cell body. A third possibility is that natural sense-antisense transcript pairs are expressed locally within dendrites or within dendritic spines, which may potentially be processed by dicer into small inhibitory RNAs (siRNAs). Indeed, we have demonstrated experimentally that a population of poly A+-bearing natural sense-antisense transcript pairs are detectably co-expressed within synaptoneurosomes (Smalheiser et al. 2008). However, because microRNAs are expressed within dendrites, where they locally regulate protein translation, we hypothesized that dicer within dendritic spines is needed to process small hairpin precursors (pre-miRs) into mature microRNAs.

\section{miRNA Precursors (pre-miRs) Are Expressed in Synaptic Fractions and Enriched at PSDs}

We employed RT-PCR methods that are able to measure microRNA precursors (both primary miRNA gene transcripts (pri-mirs) and pre-miRs) without cross-reacting with mature microRNAs. Conversely, using looped primers and Taqman probes, we were able to measure mature microRNAs without cross-reacting with precursor RNAs. Using these methods, robust expression of microRNA precursors was detected within synaptic fractions, both synaptoneurosomes and synaptosomes, at levels at or above the levels observed in forebrain tissue homogenates (Fig. 3; Lugli et al. 2008). For six miRNA precursors tested, $70-90 \%$ of the pool of miRNA precursors expressed within synaptosomes was tightly associated with the post-synaptic density fraction (Fig. 2b). In order to learn whether the precursors were primarily due to pri-miRs or pre-miRs, total RNA was size-selected, and big versus small RNA fractions were measured separately for a number of specific miRNAs. Although pri-miRs accounted for the majority of measured miRNA precursors within the total homogenate, miRNA precursor activity within the synaptic fractions appeared to consist mainly, if not exclusively, of pre-miRs (Lugli et al. 2008). Thus, we concluded that dicer and pre-miRs are both co-expressed in close proximity in association with postsynaptic densities.

\section{A Subset of Mature miRNAs Are Enriched in Synaptic Fractions}

We profiled the expression of mature miRNAs within synaptoneurosomes (SYN) using the Invitrogen NCODE microarray platform, and found that about $10 \%$ of the expressed miRNAs are significantly enriched within this synaptic fraction, whereas a somewhat smaller number are significantly depleted relative to total tissue homogenate (Lugli et al. 2008). As a group, the SYN-enriched miRNAs are biologically quite distinct from the set of miRNAs that are depleted or that show no enrichment. SYN-enriched miRNAs tend to be expressed at lower levels, to have a more discrete tissue distribution, and to include more evolutionarily recent miRNAs (i.e., those detected only in mammalian genomes), in contrast to SYN-depleted miRNAs that are predominantly expressed at high levels in many tissues, and are strongly conserved across vertebrates (some even are expressed in C. elegans).

Furthermore, for seven miRNAs, we correlated the expression of the pre-miRs and the mature miRNAs in synaptic fractions. Mature microRNAs showing high SYN enrichment exhibited significantly higher SYN enrichment of their pre-miRs as a group than the premiRs whose mature microRNAs showed low SYN enrichment (Lugli et al. 2008). This suggests that mature microRNAs present in synaptic fractions arise, at least in part, from local processing of pre-miRs within or near dendritic spines. 


\section{How Do pre-miRs Get Transported to Synaptic Regions?}

If pre-miRs are expressed in close association with post-synaptic densities, then they must have been transported stably down dendrites and within dendritic spines. However, since the mechanism of pre-miR transport has not yet been studied, at least three different models are possible. (a) pri-miRs may be transported initially to dendrites and then processed to premiRs. This is worth keeping in mind because, although no one has yet reported evidence that Drosha can exhibit a cytoplasmic localization, several proteins generally thought to be nuclear have been shown to accompany RNAs to dendrites (e.g., spliceosomal components, Glanzer et al. 2005), and both dicer and Argonaute homologs, first thought to be expressed exclusively in the cytoplasm, have been shown to have nuclear locations and functions (e.g., Lugli et al. 2005; Weinmann et al. 2009). (b) A broad population of pre-miRs may be nonselectively bound to transport complexes, but only a subset of pre-miRs become selectively stabilized against immediate processing by dicer in the cell body, or become selectively associated with postsynaptic densities. (c) A subset of pre-miRs may be specifically incorporated into transport complexes and transported down dendrites, where they account for the population of synaptically enriched mature microRNAs.

Studies are currently underway to examine these possibilities. However, it is interesting to note that the pre-miRs of SYN-enriched miRNAs differ, as a group, in their structural features as compared to SYN-depleted or non-enriched microRNAs (Smalheiser 2008b). Specifically, the pre-miRs of SYN-enriched miRNAs exhibit significantly shorter "stems", and exhibit significantly more "bulges" than do the pre-miRs of SYN-depleted or nonenriched microRNAs (see Fig. 4). The excess "bulges" comprise a single type, namely, single unpaired nucleotides on each side of the hairpin (Smalheiser 2008b). Thus, there exists a structural basis by which pre-miRs differ among each other, that may affect their interactions with cellular proteins to affect their transport or to inhibit their immediate cleavage by dicer.

\section{A Model of miRNAs in Synaptic Plasticity-I}

Based on the foregoing experimental findings, it is straightforward to elaborate upon our earlier models (Lugli et al. 2005, 2008) of how microRNAs may participate in the local modifications of protein translation that underlie plastic changes such as dendritic spine elongation and long-term potentiation.

1. A population of miRNA precursors (pre-miRs) is transported down to dendrites and "held" stably in association with postsynaptic densities.

2. Synaptic stimulation that elicits a rise in intracellular calcium beyond a certain threshold causes activation of calpain, which cleaves dicer, activates its RNAse III activity, and releases it from postsynaptic densities.

3. Local pre-miRs are processed by dicer into mature microRNAs that become associated with nearby eIF2c proteins to form RISC complexes. (Note that the core RISC component, Argonaute homolog eIF2c, is also expressed in dendritic spines; a portion of this protein is associated with postsynaptic densities and released by calpain in full-length form (Fig. 1b, 2a; Lugli et al. 2005, 2008).)

4. Loaded RISC complexes may bind to target mRNAs that are present at polyribosomes. Although most polyribosomes are located along dendritic shafts, polyribosomes are expressed within dendritic spines following stimuli that elicit LTP (e.g., Bourne et al. 2007), and both mRNAs (Suzuki et al. 2007) and elements of the protein synthesis machinery (e.g., ribosomes, EIF4E, etc.) are detected 
within dendritic spines in close proximity to postsynaptic densities (Asaki et al. 2003).

5. What happens next? Most evidence suggests that microRNAs normally inhibit translation of their targets, in neurons as in other cell types (e.g., Schratt et al. 2006; Wayman et al. 2008). Yet that might seem to imply that synaptic activity should be associated with a transient inhibition of translation, instead of the observed transient BURST of translation of synaptic mRNAs (Weiler et al. 2004). Perhaps a clue can be found from considering the emerging dual role of fragile $\mathrm{X}$ mental retardation protein (FMRP) in this situation. Whereas FMRP appears to inhibit protein translation of its targets tonically, it undergoes a transient, reversible dephosphorylation that correlates with a transient burst in translation (Narayanan et al. 2007; Kim et al. 2008). Thus, it appears that FMRP both inhibits translation under resting conditions and permits or induces translation phasically after synaptic stimulation. There is accumulating evidence in both Drosophila and mammals that FMRP exerts its actions through interactions with the microRNA pathway (reviewed in Li et al. 2008). Furthermore, recent studies in dividing non-neural cells have shown that, depending on the state of the cell cycle, a given microRNA may either inhibit or enhance translation of its targets, in a manner that probably reflects reversible modifications of the RISC-associated FMRP homolog protein FXR1 (Vasudevan et al. 2007). Regardless of how FMRP or its homologs may be implicated in this process, it is not hard to envision that synaptic microRNAs may similarly repress local translation under "resting" conditions, yet permit or enhance translation phasically following synaptic stimulation that exceeds a certain threshold.

Another conceivable scenario is suggested by the observation that partial inhibition of general protein synthesis results in enhanced translation of Arc protein (Park et al. 2008). As well, different microRNAs can compete among themselves for access to RISC (Khan et al. 2009). Either mechanism may cause an acute rise in induced microRNAs to inhibit translation of their own specific targets, leading to the facilitated translation of other nontarget mRNAs.

\section{A Model of miRNAs in Synaptic Plasticity-II}

Although many of the steps in microRNA biogenesis have been elucidated, almost nothing is yet known regarding the turnover of microRNAs within loaded RISC complexes- either those that are bound to mRNA targets or those that are not bound to any target at all. Little is known of the manner or subcellular locations at which microRNAs are degraded-are they directly cut by exonucleases (Ramachandran and Chen 2008) or is the entire RISC complex targeted for destruction, within the P body or elsewhere? siRNA-mediated gene silencing can be effectively maintained within neurons for weeks (Omi et al. 2004), suggesting that loaded RISC can be extremely stable under at least some conditions.

Given these uncertainties, it is premature to make a serious model regarding the long-term role of microRNAs within dendritic spines. However, if loaded RISC (in association with synaptic mRNA targets) is stable for days to weeks, then it may serve as a persistent local regulator of long-term synaptic efficacy within a previously activated synapse. And, if loaded RISC that is NOT bound to targets is stable for at least hours to days, then it may serve as a "trap" to bind mRNAs that are being transported down dendrites, and route them to previously activated synapses. Thus, it is intriguing to wonder if microRNAs may also be playing roles in synaptic tagging and capture (Martin and Kosik 2002; Barco et al. 2008). 


\section{Unanswered Questions}

Our model makes a number of testable predictions and suggests further questions to ask. For example, we expect that pre-miRs will be found to be associated with transport protein complexes at least in neurons (if not in non-neural cells), though it has not yet been ruled out that primary miRNA gene transcripts are transported and then processed locally to pre-miRs. It is not clear whether transport of miRNA precursors should be a selective process, nor whether (like transport of synaptic mRNAs) it may be stimulated by synaptic activity. We still need to demonstrate experimentally that synaptic pre-miRs show a burst of processing to mature miRNAs upon synaptic activation, and that this permits or enhances protein translation of specific targets. Park and Tang (2008) showed that chemical LTP in hippocampal slices causes the up-regulation of nearly all detectable microRNAs after 15 min, which is more dramatic than one might have expected, yet is consistent with the occurrence of an activity-dependent burst of microRNA formation. We have also identified significant up-regulation of numerous microRNAs, which occurs in hippocampus during an early stage of olfactory discrimination training in mice (Smalheiser et al. manuscript submitted). However, it is not known yet whether microRNAs regulate specific aspects of LTP induction or maintenance, or specific stages in learning and memory.

Given the importance of phosphorylation in regulating multiple proteins involved in protein translation and synaptic efficacy, it is important to examine the possible regulatory role of phosphorylation not only of FMRP, but also of dicer co-factors (TRBP and PACT) and RISC (eIF2C) (Zeng et al. 2008). As well, it is important to learn how stable loaded RISC complexes are, both when bound and when unbound to specific mRNA targets, and whether microRNAs are regulated not only via their processing but also via their selective turnover.

Finally, one wonders whether microRNAs are the only tiny RNAs that have roles in regulating synaptic plasticity, either acting within the cell body or locally within dendrites. The observation that many natural sense-antisense transcript pairs are co-expressed within adult brain (Kiyosawa et al. 2005) and within synaptic fractions (Smalheiser et al. 2008) raises anew the question whether these may be processed to siRNAs and thus participate in long-term regulation of neuronal gene expression (Smalheiser et al. 2001). Though examples of normal gene regulation by siRNAs are still few and far between, recent studies have obtained evidence for the existence of endogenous siRNAs in C. elegans, Drosophila, and mammalian cells (reviewed in Kim et al. 2009), so an examination of neurons is certainly warranted-bearing in mind that, unlike microRNAs, siRNAs may exhibit modifications on their $3^{\prime}$-ends that make them difficult to detect using some current sequencing methods.

\section{Conclusion}

The studies reviewed here emphasize the importance of microRNAs in regulating synaptic plasticity in the normal adult brain, and focus attention on novel cellular mechanisms (e.g., miRNA precursor transport and local calcium-dependent processing) that have not previously gained much attention among biologists. Numerous neuropsychi-atric disorders are thought to involve abnormalities in synaptic plasticity and have been associated with micro-RNA changes, for example, autism (Abu-Elneel et al. 2008), fragile $X$ mental retardation syndrome (Xu et al. 2008), Rett syndrome (Nomura et al. 2008), depression (Duman 2002; Smalheiser et al. 2009), and schizophrenia (Hansen et al. 2007; Perkins et al. 2007; Beveridge et al. 2008). Therapeutic agents such as mood stabilizers, which appear to influence synaptic plasticity, may act upon miRNAs and proteins of the microRNA pathway (Zhou et al. 2009). Therefore, we suggest that these novel mechanisms should also be considered when investigating the manner in which microRNAs may be implicated in the pathogenesis of human brain diseases. 


\section{Acknowledgments}

We are grateful to Drs. John Larson, Yogesh Dwivedi, and Vetle Torvik for their expert collaborations. Our research was supported by NIH grants DA15450, MH81099, DC 05793, and LM07292 and by the Stanley Medical Research Institute. The contents of this article are solely the responsibility of the authors and do not necessarily represent the official views of NIH.

\section{References}

Abu-Elneel K, Liu T, Gazzaniga FS, Nishimura Y, Wall DP, Geschwind DH, et al. Heterogeneous dysregulation of microRNAs across the autism spectrum. Neurogenetics. 2008; 9:153-161. [PubMed: 18563458]

Asaki C, Usuda N, Nakazawa A, Kametani K, Suzuki T. Localization of translational components at the ultrami-croscopic level at postsynaptic sites of the rat brain. Brain Research. 2003; 972:168176. [PubMed: 12711090]

Ashraf SI, McLoon AL, Sclarsic SM, Kunes S. Synaptic protein synthesis associated with memory is regulated by the RISC pathway in Drosophila . Cell. 2006; 124:191-205. [PubMed: 16413491]

Barco A, Lopez de Armentia M, Alarcon JM. Synapse-specific stabilization of plasticity processes: The synaptic tagging and capture hypothesis revisited 10 years later. Neuroscience and Biobehavioral Reviews. 2008; 32:831-851. [PubMed: 18281094]

Beveridge NJ, Tooney PA, Carroll AP, Gardiner E, Bowden N, Scott RJ, et al. Dysregulation of miRNA $181 \mathrm{~b}$ in the temporal cortex in schizophrenia. Human Molecular Genetics. 2008; 17:11561168. [PubMed: 18184693]

Bourne JN, Sorra KE, Hurlburt J, Harris KM. Polyribosomes are increased in spines of CA1 dendrites $2 \mathrm{~h}$ after the induction of LTP in mature rat hippocampal slices. Hippocampus. 2007; 2007(17):1-4. [PubMed: 17094086]

Dincbas-Renqvist V, Pe'pin G, Rakonjac M, Plante I, Ouellet DL, Hermansson A, et al. Human Dicer C-terminus functions as a 5-lipoxygenase binding domain. Biochimica et Biophysica Acta. 2009; 1789:99-108. [PubMed: 19022417]

Duman RS. Pathophysiology of depression: the concept of synaptic plasticity. European Psychiatry. 2002; 17(Suppl 3):306-310. [PubMed: 15177086]

Eis PS, Tam W, Sun L, Chadburn A, Li Z, Gomez MF, et al. Accumulation of miR-155 and BIC RNA in human B cell lymphomas. Proceedings of the National Academy of Sciences of the United States of America. 2005; 102:3627-3632. [PubMed: 15738415]

Glanzer J, Miyashiro KY, Sul JY, Barrett L, Belt B, Haydon P, et al. RNA splicing capability of live neuronal dendrites. Proceedings of the National Academy of Sciences of the United States of America. 2005; 102:16859-16864. [PubMed: 16275927]

Hansen T, Olsen L, Lindow M, Jakobsen KD, Ullum H, Jonsson E, et al. Brain expressed microRNAs implicated in schizophrenia etiology. PLoS ONE. 2007; 2:e873. [PubMed: 17849003]

John B, Enright AJ, Aravin A, Tuschl T, Sander C, Marks DS. Human MicroRNA targets. PLoS Biology. 2004; 2:e363. [PubMed: 15502875]

Khan AA, Betel D, Sander C, Leslie CS, Marks DS. Nature Biotechnology. 2009 (in press).

Kim VN, Han J, Siomi MC. Biogenesis of small RNAs in animals. Nature Reviews. Molecular Cell Biology. 2009; 10:126-139.

Kim SH, Markham JA, Weiler IJ, Greenough WT. Aberrant early-phase ERK inactivation impedes neuronal function in fragile X syndrome. Proceedings of the National Academy of Sciences of the United States of America. 2008; 105:4429-4434. [PubMed: 18332424]

Kiyosawa H, Mise N, Iwase S, Hayashizaki Y, Abe K. Disclosing hidden transcripts: mouse natural sense-antisense transcripts tend to be poly(A) negative and nuclear localized. Genome Research. 2005; 15:463-474. [PubMed: 15781571]

Klein ME, Lioy DT, Ma L, Impey S, Mandel G, Goodman RH. Homeostatic regulation of MeCP2 expression by a CREB-induced microRNA. Nature Neuroscience. 2007; 10:1513-1514.

Kosik KS. The neuronal microRNA system. Nature Reviews. Neuroscience. 2006; 7:911-920. 
Kye MJ, Liu T, Levy SF, Xu NL, Groves BB, Bonneau R, et al. Somatodendritic microRNAs identified by laser capture and multiplex RT-PCR. RNA. 2007; 13:1224-1234. [PubMed: 17592044]

Li Y, Lin L, Jin P. The microRNA pathway and fragile X mental retardation protein. Biochimica et Biophysica Acta. 2008; 1779:702-705. [PubMed: 18687414]

Lugli G, Larson J, Martone ME, Jones Y, Smalheiser NR. Dicer and eIF2c are enriched at postsynaptic densities in adult mouse brain and are modified by neuronal activity in a calpain-dependent manner. Journal of Neurochemistry. 2005; 94:896-905. [PubMed: 16092937]

Lugli G, Torvik VI, Larson J, Smalheiser NR. Expression of microRNAs and their precursors in synaptic fractions of adult mouse forebrain. Journal of Neurochemistry. 2008; 106:650-661. [PubMed: 18410515]

Martin KC, Kosik KS. Synaptic tagging-Who's it? Nature Reviews. Neuroscience. 2002; 3:813-820.

Narayanan U, Nalavadi V, Nakamoto M, Pallas DC, Ceman S, Bassell GJ, et al. FMRP phosphorylation reveals an immediate-early signaling pathway triggered by group I mGluR and mediated by PP2A. Journal of Neuroscience. 2007; 27:14349-14357. [PubMed: 18160642]

Nomura T, Kimura M, Horii T, Morita S, Soejima H, Kudo S, et al. MeCP2-dependent repression of an imprinted miR-184 released by depolarization. Human Molecular Genetics. 2008; 17:11921199. [PubMed: 18203756]

Omi K, Tokunaga K, Hohjoh H. Long-lasting RNAi activity in mammalian neurons. FEBS Letters. 2004; 558:89-95. [PubMed: 14759522]

Park S, Park JM, Kim S, Kim JA, Shepherd JD, Smith-Hicks CL, et al. Elongation factor 2 and fragile $\mathrm{X}$ mental retardation protein control the dynamic translation of Arc/Arg3.1 essential for mGluRLTD. Neuron. 2008; 59:70-83. [PubMed: 18614030]

Park CS, Tang SJ. Regulation of microRNA Expression by Induction of Bidirectional Synaptic Plasticity. Journal of Molecular Neuroscience. 2008; 38:50-66. [PubMed: 18998061]

Perkins DO, Jeffries CD, Jarskog LF, Thomson JM, Woods K, Newman MA, et al. microRNA expression in the prefrontal cortex of individuals with schizophrenia and schizo-affective disorder. Genome Biology. 2007; 8:R27. [PubMed: 17326821]

Provost P, Dishart D, Doucet J, Frendewey D, Samuelsson B, Radmark O. Ribonuclease activity and RNA binding of recombinant human Dicer. EMBO Journal. 2002; 21:5864-5874. [PubMed: 12411504]

Ramachandran V, Chen X. Degradation of microRNAs by a family of exoribonucleases in Arabidopsis . Science. 2008; 321:1490-1492. [PubMed: 18787168]

Schratt GM, Tuebing F, Nigh EA, Kane CG, Sabatini ME, Kiebler M, et al. A brain-specific microRNA regulates dendritic spine development. Nature. 2006; 439:283-289. [PubMed: 16421561]

Smalheiser NR. Regulation of mammalian microRNA processing and function by cellular signaling and subcellular localization. Biochimica et Biophysica Acta. 2008a; 1779:678-681. [PubMed: 18433727]

Smalheiser NR. Synaptic enrichment of microRNAs in adult mouse forebrain is related to structural features of their precursors. Biology Direct. 2008b; 3:44. [PubMed: 18957138]

Smalheiser NR, Lugli G, Lenon AL, Larson J.

Smalheiser NR, Lugli G, Rizavi HS, Turecki D, Torvik VI, Dwivedi Y. 2009

Smalheiser NR, Lugli G, Torvik VI, Mise N, Ikeda R, Abe K. Natural antisense transcripts are coexpressed with sense mRNAs in synaptoneurosomes of adult mouse forebrain. Neuroscience Research. 2008; 62:236-239. [PubMed: 18812194]

Smalheiser NR, Manev H, Costa E. RNAi and brain function: Was McConnell on the right track? Trends in Neurosciences. 2001; 24:216-218. [PubMed: 11250005]

Suzuki T, Tian QB, Kuromitsu J, Kawai T, Endo S. Characterization of mRNA species that are associated with postsynaptic density fraction by gene chip microarray analysis. Neuroscience Research. 2007; 57:61-85. [PubMed: 17049655]

Vasudevan S, Tong Y, Steitz JA. Switching from repression to activation: microRNAs can up-regulate translation. Science. 2007; 318:1931-1934. [PubMed: 18048652] 
Velleca MA, Wallace MC, Merlie JP. A novel synapse-associated noncoding RNA. Molecular and Cellular Biology. 1994; 14:7095-7104. [PubMed: 7523860]

Vo N, Klein ME, Varlamova O, Keller DM, Yamamoto T, Goodman RH, et al. cAMP-response element binding protein-induced microRNA regulates neuronal morphogenesis. Proceedings of the National Academy of Sciences of the United States of America. 2005; 102:16426-16431. [PubMed: 16260724]

Wayman GA, Davare M, Ando H, Fortin D, Varlamova O, Cheng HY, et al. An activity-regulated microRNA controls dendritic plasticity by down-regulating p250GAP. Proceedings of the National Academy of Sciences of the United States of America. 2008; 105:9093-9098. [PubMed: 18577589]

Weiler IJ, Spangler CC, Klintsova AY, Grossman AW, Kim SH, Bertaina-Anglade V, et al. Fragile X mental retardation protein is necessary for neurotransmitter-activated protein translation at synapses. Proceedings of the National Academy of Sciences of the United States of America. 2004; 101:17504-17509. [PubMed: 15548614]

Weinmann L, Ho“ck J, Ivacevic T, Ohrt T, Mu“tze J, Schwille P, et al. Importin 8 is a gene silencing factor that targets argonaute proteins to distinct mRNAs. Cell. 2009; 136:496-507. [PubMed: 19167051]

Wu J, Xie X. Comparative sequence analysis reveals an intricate network among REST, CREB and miRNA in mediating neuronal gene expression. Genome Biology. 2006; 7:R85. [PubMed: 17002790]

Xu XL, Li Y, Wang F, Gao FB. The steady-state level of the nervous-system-specific microRNA-124a is regulated by dFMR1 in Drosophila. Journal of Neuroscience. 2008; 28:11883-11889. [PubMed: 19005053]

Zeng Y, Sankala H, Zhang X, Graves PR. Phosphorylation of Argonaute 2 at serine- 387 facilitates its localization to processing bodies. Biochemical Journal. 2008; 413:429-436. [PubMed: 18476811]

Zhang H, Kolb FA, Brondani V, Billy E, Filipowicz W. Human Dicer preferentially cleaves dsRNAs at their termini without a requirement for ATP. EMBO Journal. 2002; 21:5875-5885. [PubMed: 12411505]

Zhou R, Yuan P, Wang Y, Hunsberger JG, Elkahloun A, Wei Y, et al. Evidence for selective microRNAs and their effectors as common long-term targets for the actions of mood stabilizers. Neuropsychopharmacology. 2009; 34:1395-1405. [PubMed: 18704095] 

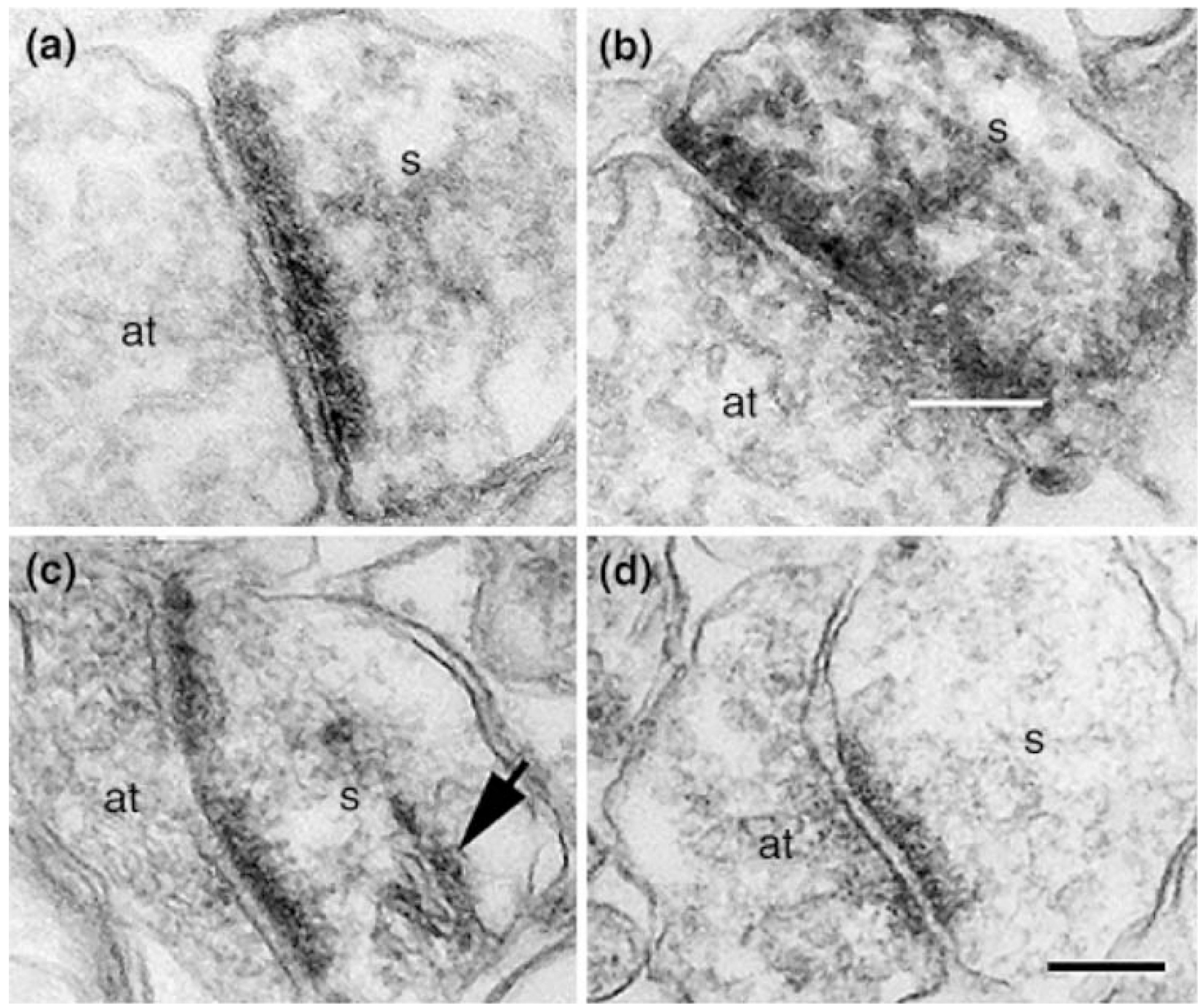

Fig. 1.

Electron microscopic localization of dicer and eIF2c in dendritic spines in cortex and hippocampal area CA1. The distribution of dicer $(\mathrm{a}, \mathrm{c})$ tended to be more discrete than eIF2c (b). In the cortical spine in (c), a deposit of label can be seen surrounding the spine apparatus (arrow). Postsynaptic densities were heavily labeled for both proteins, much greater than the slight contrast due to heavy metal staining that can be seen in tissue labeled without the primary antibody (d). $s$ spine head; at axon terminal. Scale bar $=200 \mathrm{~nm}$. Reprinted from Lugli et al. (2005) by permission of Journal of Neurochemistry 


\section{(a)}

\section{T S2 Sy Ss Sp}

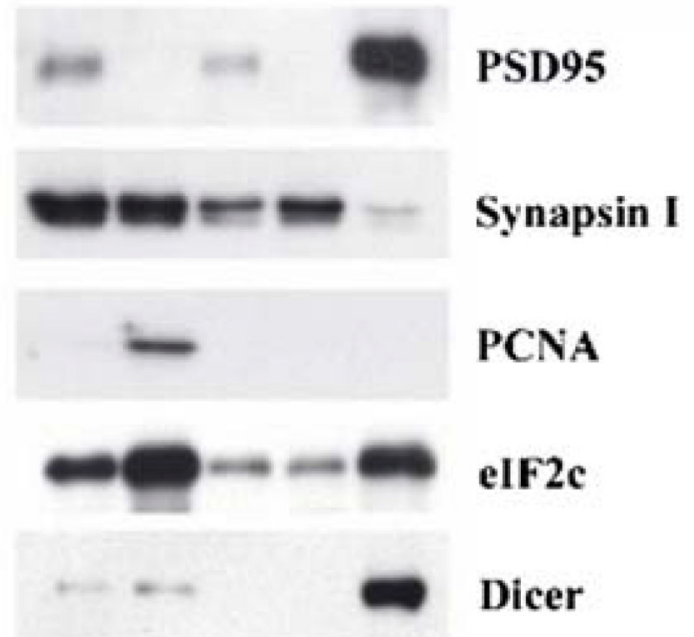

(b)

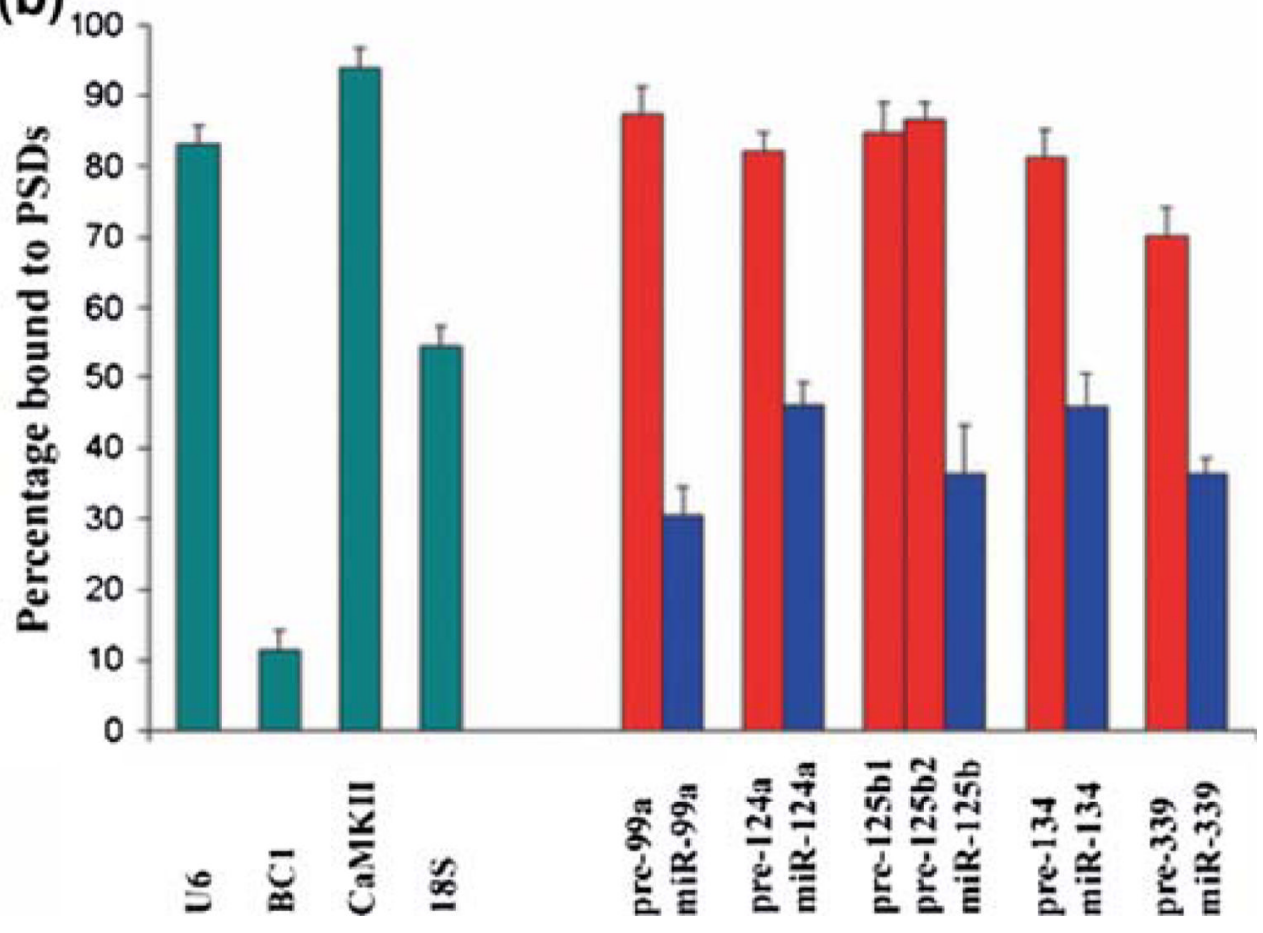

Fig. 2.

Distribution of synaptosomal proteins and RNAs in soluble versus PSD fractions after extraction with Triton X-100. a Immuno-blotting. Total forebrain homogenate (T) was processed to obtain a soluble cytoplasmic fraction (S2) and a synaptosomal fraction (Sy) that was then lysed with $1 \%$ Triton X-100 to yield soluble (Ss) and PSD fractions (Sp). Equal amounts of protein were loaded and blotted for different antibodies as indicated. The dicer antibody was chicken polyclonal anti-dicer (Lugli et al. 2005). b qRT-PCR measurements of RNAs. Total RNA was prepared from Ss and $\mathrm{Sp}$ fractions and measured. The Sp/Sy ratio was calculated for three independent preps and is plotted as shown. microRNA precursors 
are shown in red, mature microRNAs in blue, and other RNAs in green. Reprinted from Lugli et al. (2008) by permission of Journal of Neurochemistry 


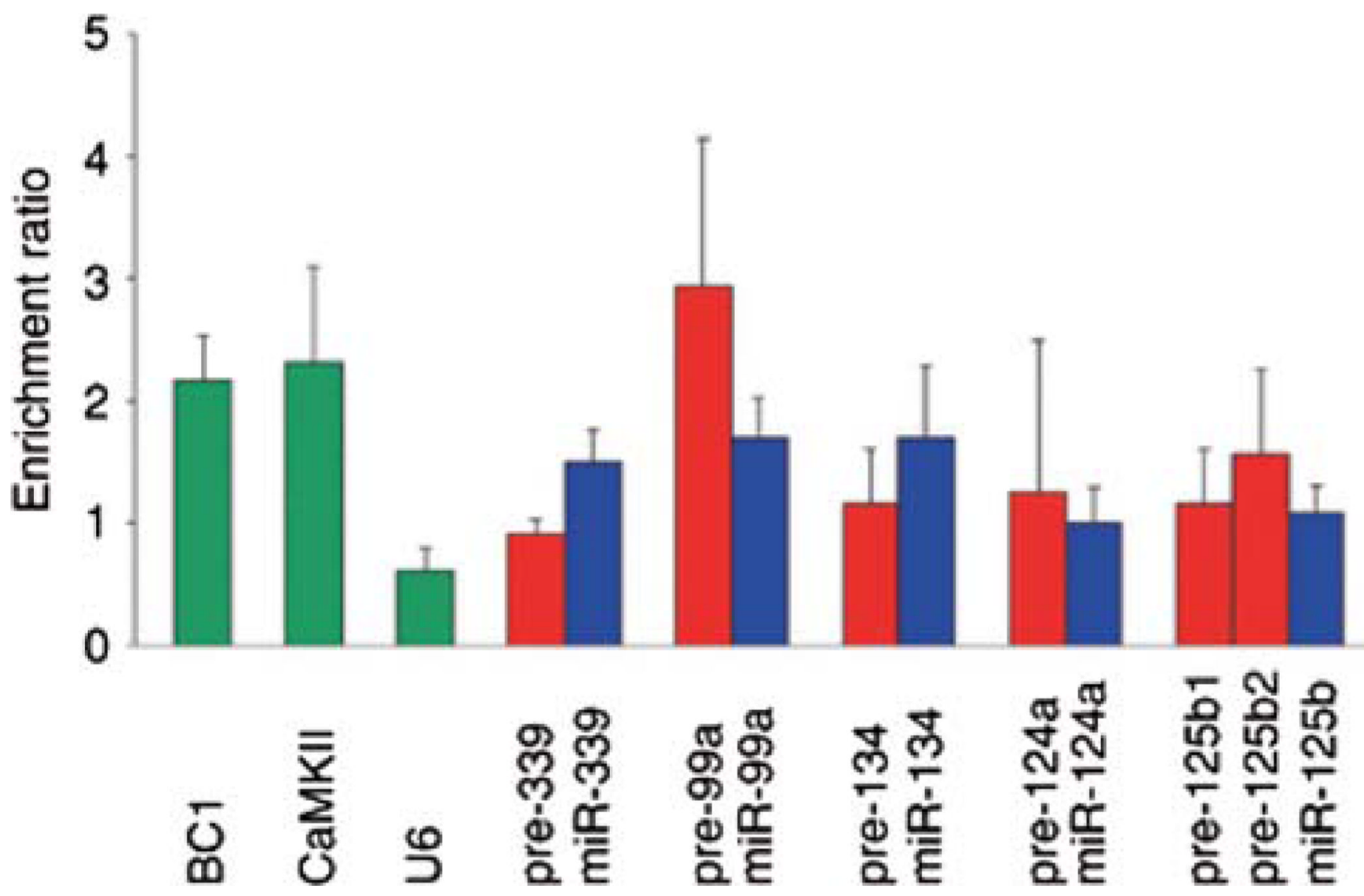

Fig. 3.

Enrichment ratio (synaptoneurosomes/total homogenate) of various RNAs as measured by qRT-PCR. Data represent the average of three independent preps. microRNA precursors are shown in red, mature microRNAs in blue, and other RNAs in green. Reprinted from Lugli et al. (2008) by permission of Journal of Neurochemistry 


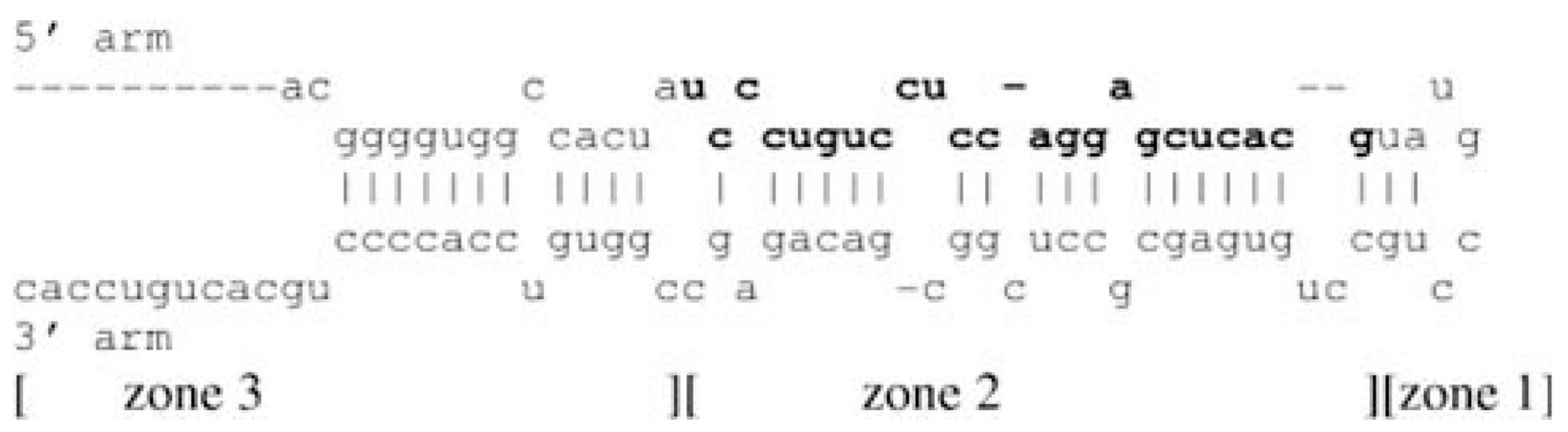

Fig. 4.

Diagram of a microRNA small hairpin precursor (pre-miR). Shown is pre-miR-339. Zone 1 comprises the loop, zone 2 includes the region giving rise to the mature mir-339 sequence (shown in bold), and zone 3 includes sequences (if any) below the mature microRNA.

Regions of bases connected by bonds (including G:U bonds) are referred to as stems, and opposing bases not connected by bonds are referred to as bulges. Reprinted from Smalheiser (2008b) 